\title{
Papillary Squamotransitional Cell Carcinoma Cervix: A Rare Variant
}

\author{
${ }^{1}$ Monika Anant, ${ }^{2}$ Amrita Singh, ${ }^{3}$ Vandita Y Singh
}

\begin{abstract}
Papillary squamotransitional cell carcinoma (PSTCC) is a lesser known histological subtype of the most common type of cervical "squamous cell carcinoma (SCC)." Papillary carcinomas of the uterine cervix with transitional or squamous differentiation often resemble transitional cell carcinomas of the urinary tract.In the literature, PSTCC is underdiagnosed, has late presentation, is more common in postmenopausal age group, and has a late recurrence. Here we report a case of a 52-year-old lady presenting with postmenopausal bleeding, whose cytological evaluation reported atypical squamous cells of undetermined significance (ASCUS) and cervical biopsy as high-grade squamous intraepithelial lesion (HSIL). She underwent laparoscopic hysterectomy to be diagnosed with PSTCC mixed type. With an 18-month follow-up, she has shown no locoregional recurrence. A high index of suspicion on the part of the clinician and an awareness of PSTCC subtype of cancer cervix by the histopathologist are required to make an accurate diagnosis.
\end{abstract}

Keywords: Cancer, Cervix, Papillary, Squamous, Transitional.

How to cite this article: Anant M, Singh A, Singh VY. Papillary Squamotransitional Cell Carcinoma Cervix: A Rare Variant. J South Asian Feder Menopause Soc 2017;5(2):142-144.

Source of support: Nil

Conflict of interest: None

Date of received: 3 August 2017

Date of acceptance: 17 Septemer 2017

Date of publication: December 2017

\section{INTRODUCTION}

All over the globe, the most common type of reported cervical cancer is SCC keratinizing or nonkeratinizing. Lesser common ones are those having papillary components in squamous cells, which can be in three different forms: (1) Predominantly squamous type, (2) mixed

\footnotetext{
${ }^{1-3}$ Assistant Professor

1,2Department of Obstetrics \& Gynecology, All India Institute of Medical Sciences, Patna, Bihar, India

${ }^{3}$ Department of Pathology and Laboratory Medicine, All India Institute of Medical Sciences, Patna, Bihar, India

Corresponding Author: Monika Anant, Assistant Professor Department of Obstetrics \& Gynecology, All India Institute of Medical Sciences, Patna, Bihar, India, Phone: +917764946249 e-mail: drmonika.anant@gmail.com
}

squamotransitional type (PSTCC), or (3) predominantly transitional type. ${ }^{1}$

Papillary squamotransitional cell carcinoma is a lesser known histological subtype. These tumors have a distinguishing surface papillary growth pattern and can be deeply invading. The PSTCC of the uterine cervix is considered to be associated with an aggressive biological behavior. They tend to recur locally or even metastasize over a longer period of time.,3

It has also been reported to present at a more advanced stage, despite the histopathological evidence suggesting a superficial or early invasive lesion. ${ }^{4}$

It becomes necessary to separately identify from the commoner and benign varieties of the cervical papillary lesions like condyloma acuminata and squamous papillomas because of its potentially aggressive biological course.

The recognition of PSTCC of the cervix is important despite being less common, to demarcate its clinicopathological features and progress of disease. A high index of suspicion on the part of the clinician and an awareness of PSTCC by the pathologist are required to make an accurate diagnosis.

Here, we report a case of a postmenopausal lady with PSTCC of cervix.

\section{CASE REPORT}

A 52-year-old, parity and lining tissue $\left(\mathrm{P}_{4} \mathrm{~L}_{3}\right)$, postmenopausal lady presented to our outpatient department with complaints of nonodorous, purulent vaginal discharge since 1 year. She had her menopause 6 years back; although there was no history of postmenopausal bleeding, she had discharge per vaginum on and off since last 1 year. She was recently a diagnosed case of diabetes mellitus and had been started on oral hypoglycemic agents. She had no history of oral contraceptive use; partner was single with no exposure to sexually transmitted diseases. She had not undergone cervical cancer screening anytime in her reproductive lifespan. Her general physical examination was normal as per her age. In systemic examination as well as in abdominal examination, no abnormalities were detected.

As a part of the gynecological examination, cervix on speculum examination looked unhealthy with congestions and irregularity, although no obvious growth was seen. Thin mucopurulent discharge was also present. Vagina 


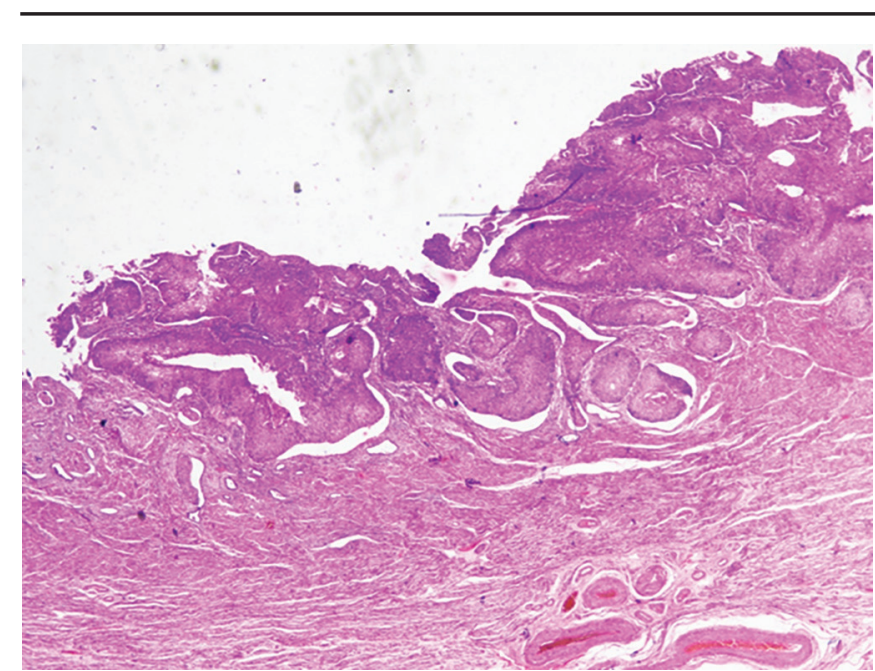

Fig. 1: Scanner view reveals small exophytic papillary structures with delicate fibrovascular cores and invasive component in the form of nests. H\&E, 40X $(4 X \times 10)$

appeared to be healthy. Uterus was normal size, nontender, and mobile with nonpalpable adenexa and parametrium. However, bleeding from cervix on manipulation was seen. Cervical Papanicolaou (Pap) smear was taken.

Cervical Pap smear testing report came out with negative for intraepithelial cell or malignancy along with features of inflammatory cervical smear with presence of atypical squamous cells of undetermined significance (ASCUS). In view of the report, colposcopy-directed cervical biopsy was done and resultant report was "suspicious of squamous cell carcinoma." Invasion of lesion could not be commented upon.

Loop electrosurgical excision procedure of cervix was planned to get a proper depth of biopsy specimen. Histopathology reported HSILs and, once again, margin invasion could not be commented upon due to cautery effect.

The magnetic resonance imaging could not be done due to monetary concerns. Hematology and other laboratory tests including serum electrolytes and renal and hepatic function tests were found within normal limits. Surgical management was formulated for a suspicious cancer cervix with no obvious growth (stage 1 a1) in this postmenopausal woman by laparoscopic hysterectomy and bilateral salpingo-oophorectomy.

Intraoperatively, uterus and bilateral tubes and ovaries were normal. Cut section of the specimen showed pale yellowish uterine body and cervix, with no grossly visible tumor or lesion.

Histopathology reported it to be a PSTCC (Figs 1 and 2) (mixed type) $\left(\mathrm{pT}^{\mathrm{b}} \mathrm{b}\right)\left(\mathrm{pN}_{\mathrm{X}}\right)$, with tumor extension into uterine corpus. However, serosal layer was free of tumor (closest distance $0.1 \mathrm{~cm}$ away from serosal surface in lower uterine segment). Another finding was of cervical intraepithelial neoplasia grade III (CIN III) at cervical resection margin. However, invasive carcinoma was lying $0.4 \mathrm{~cm}$ away from new cervical resection margin.

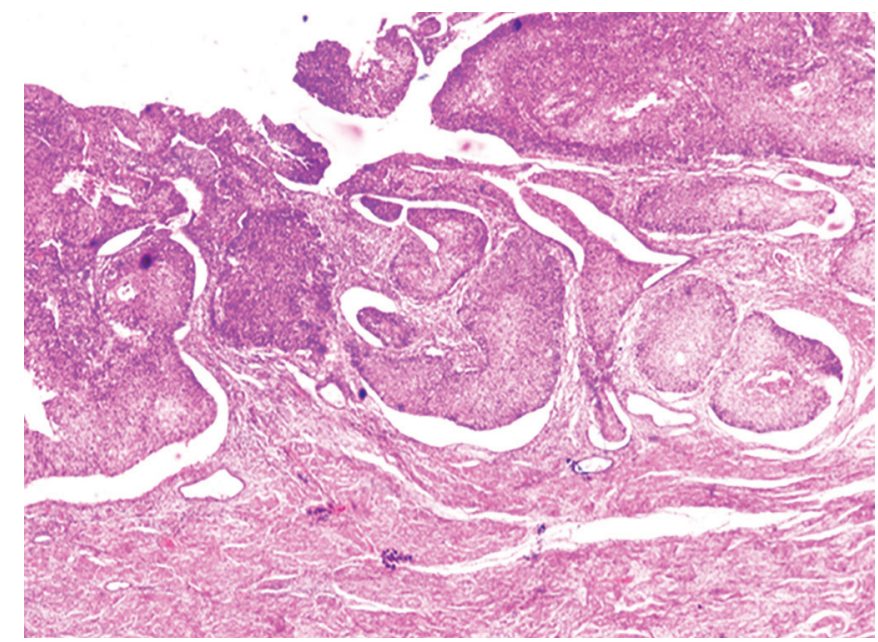

Fig. 2: Higher magnification reveals the neoplastic cells lining the papillae and the invasive nests with features of both squamous and transitional epithelial cells. H\&E, 200X (20X×10)

The patient was referred to a center for oncology where she underwent six cycles of radiation therapy (teletherapy), which was an overtreatment for her stage of cancer cervix. She is healthy and asymptomatic and free of tumor in 18 months of her follow-up.

\section{DISCUSSION}

The most common subtypes of SCC of the uterine cervix are the keratinizing and nonkeratinizing (nonpapillary) variants. ${ }^{5}$

Papillary growth pattern is recognized in some unusual SCC of the cervix, the verrucous carcinoma, the condylomatous (warty) squamous carcinoma, and the (squamo) transitional cell carcinoma, which contain both true squamous and transitional cells in combination..$^{1-3,6}$ The PSTCC is the latest subtype to be recognized and described.

Recognition of these variants is important as the course of disease progression and its further management differ among the histologic subtypes. ${ }^{7}$

Papillary SCC of cervix and its histological subtypes are considered as separate entities. This fact is supported by the study of Randall et al ${ }^{3}$ and Koenig et al. ${ }^{6}$ Randall et al in their study of nine cases suggested that papillary SCC of the cervix should be considered a distinct clinicopathological entity, separate from verrucous carcinoma.

Koenig et al studied 32 cases of PSTCC cervix. They divided them into three groups: (1) Predominantly squamous $(28.1 \%)$, (2) mixed squamous and transitional (50\%), and (3) predominantly transitional (21.9\%). Histopathologically papillary architecture with fibrovascular cores lined by multilayered, atypical epithelium resembling high-grade squamous intraepithelial neoplasia of the cervix was found in all the cases. They concluded that papillary SCC of the cervix and its subtypes are distinct clinicopathological entities with a morphologic spectrum. 
Brinck et $\mathrm{al}^{8}$ in their study of three cases supported the hypothesis that papillary SCCs (unlike verrucous carcinoma) are similar with regard to risk factors to squamotransitional and condylomatous carcinoma.

The human papillomavirus (HPV) may play an etiologic role in some of these tumors; and papillary SCC is the only subtype among squamous / (squamo)transitional carcinomas, i.e., associated with high-risk HPV infection in the absence of HPV-related histopathologic alterations. It reaffirms relation between HPV type and clinical outcome in variants of SCC with papillary features. ${ }^{8}$

Although it is a known entity for the last three decades, difficulties are encountered in the correct histological diagnosis and staging of this malignancy. In their study, $\mathrm{Ng}^{9}$ concluded that although PSTCC has a distinctive appearance in thin-layer cytologic preparations with predominance of bland-looking basaloid cells or HSIL cells, there is underdiagnosis of the subtype due to scantiness of tumor diathesis and carcinoma cells.

Al-Nafussi and Al-Yusif, ${ }^{4}$ in their study, concluded that papillary SCC has a propensity for late metastasis and local recurrence despite the fact that histologically it could be misinterpreted as CIN3 with a papillary configuration or as a squamous papilloma.

Does cervix have transitional cells or do these cells come from the urinary tract cancers?

Cervical transitional (SCC) cells have different staining patterns $(\mathrm{CK} 7+/ \mathrm{CK} 20)$ than the transitional cell tumors of the urinary tract (CK7+/CK20+) and SCCs of the cervix. ${ }^{10,11}$ This differentiates urinary tract metastasis from transitional cell variants of SCC.

The knowledge about this important histological subtype, PSTCC, is required by both gynecologist and pathologist to differentiate it from a more benign course of other subtypes like verrucous or papillary squamous, because of its late recurrence and metastasis.

\section{REFERENCES}

1. Kurman JR, Ellenson LH, Ronnett BM. Blaustein's pathology of the female genital tract 6th ed. New York: Springer; 2011: pp. 271-272.

2. Albores-Saavedra J, Young RH. Transitional cell neoplasms (carcinomas and inverted papillomas) of the uterine cervix. A report of five cases. Am J Surg Pathol 1995 Oct;19(10): 1138-1145.

3. Randall ME, Andersen WA, Mills SE, Kim JC. Papillary squamous cell carcinoma of the uterine cervix: a clinicopathologic study of nine cases. Int J Gynecol Pathol 1986;5(1):1-10.

4. Al-Nafussi AI, Al-Yusif R. Papillary squamo transitional cell carcinoma of the uterine cervix: an advanced stage disease despite superficial location: report of two cases and review of the literature. Eur J Gynaecol Oncol 1998;19(5): 455-457.

5. Scully RE, Bonfiglio TA, Kurman RJ, SilverbergSG, WilkinsonEJ. Histological typing of female genital tract tumours, 2nd ed. Berlin: Springer-Verlag; 1994.

6. Koenig C, Turnicky RP, Kankam CF, Tavassoli FA. Papillary squamotransitional cell carcinoma of the cervix: a report of 32 cases. Am J Surg Pathol 1997 Aug;21(8):915-921.

7. Patridge EE, Murad T, Shingleton HM, Austin JM, Hatch KD. Verrucous lesions of the female genitalia. I. Giant condylomata. Am J Obstet Gynecol 1980;137:412-418.

8. Brinck U, Jakob C, Bau O, Fuzesi L. Papillary squamous cell carcinoma of the uterine cervix: report of three cases and a review of its classification. Int J Gynaecol Pathol 2000 Jul;19(3): 231-235.

9. Ng WK. Thin-layer (liquid-based) cytologic findings of papillary squamotransitional cell carcinoma of the cervix: review of cases over a 4-year period with emphasis on potential diagnostic pitfalls. Acta Cytol 2003;47:141-147.

10. Lininger RA, Ashfaq R, AlboresSaavedra J, Tavassoli FA. Transitional cell carcinoma of the endometrium and endometrial carcinoma with transitional cell differentiation. Cancer 1997 May;79(10):1933-1943.

11. Soslow RA, Rouse RV, Hendrickson MR, Silva EG, Longacre TA. Transitional cell neoplasms of the ovary and urinary bladder: a comparative immunohistochemical analysis. Int J Gynecol Pathol 1996 Jul;15(3):257-265. 